\title{
Éruption papulosquameuse avec symptômes oculaires dus à la syphilis
}

\author{
Marcus G. Tan MD, Mark G. Kirchhof MD PhD
}

- Citation : CMAJ 2020 December 7;192:E1727. doi : 10.1503/cmaj.200820-f

Voir la version anglaise de l'article ici : www.cmaj.ca/lookup/doi/10.1503/cmaj.200820

$\mathbf{U}$ ne femme de 42 ans n'ayant pas d'autres problèmes de santé s'est présentée au service des urgences avec une fièvre subjective, un mal de tête s'aggravant, une vision floue, une photophobie, des nausées et un épiphora (figure $1 \mathrm{~A}$ ), symptômes qui persistaient depuis 1 semaine. À l'examen physique, la patiente était afébrile, mais présentait une éruption papulosquameuse généralisée asymptomatique (figures 1B et 1C). L'éruption était apparue plusieurs semaines auparavant, mais la patiente n'avait pas cherché à consulter un professionnel en raison de l'absence de symptômes. L'examen à la lampe à fente semblait indiquer la présence d'une uvéite antérieure. Deux mois auparavant, la patiente avait reçu des résultats négatifs au dépistage de la syphilis, du VIH, de la gonorrhée et de la chlamydia 1 semaine après avoir eu une relation sexuelle non protégée avec un nouveau partenaire.

Une biopsie cutanée à l'abdomen a révélé une dégénérescence vacuolaire de la basale accompagnée d'infiltrats inflammatoires mixtes (plasmocytes, lymphocytes et histiocytes) superficiels et mésodermiques. Une coloration immunohistochimique a révélé la présence de spirochètes. Les résultats de l'examen hématologique de base étaient normaux. Un dépistage de la syphilis par épreuve de chimiluminescence et test rapide de la réagine plasmatique a donné un résultat positif, confirmé par un test d'agglutination passive de Treponema pallidum.

Nous avons posé un diagnostic de syphilis secondaire avec neurosyphilis oculaire. Comme la patiente avait une allergie à la pénicilline, nous avons prescrit un régime de doxycycline orale de 28 jours (100 mg, 2 fois par jour). Ses symptômes constitutionnels et oculaires se sont résorbés en 1 semaine, et l'éruption avait presque complètement disparu après 1 mois.

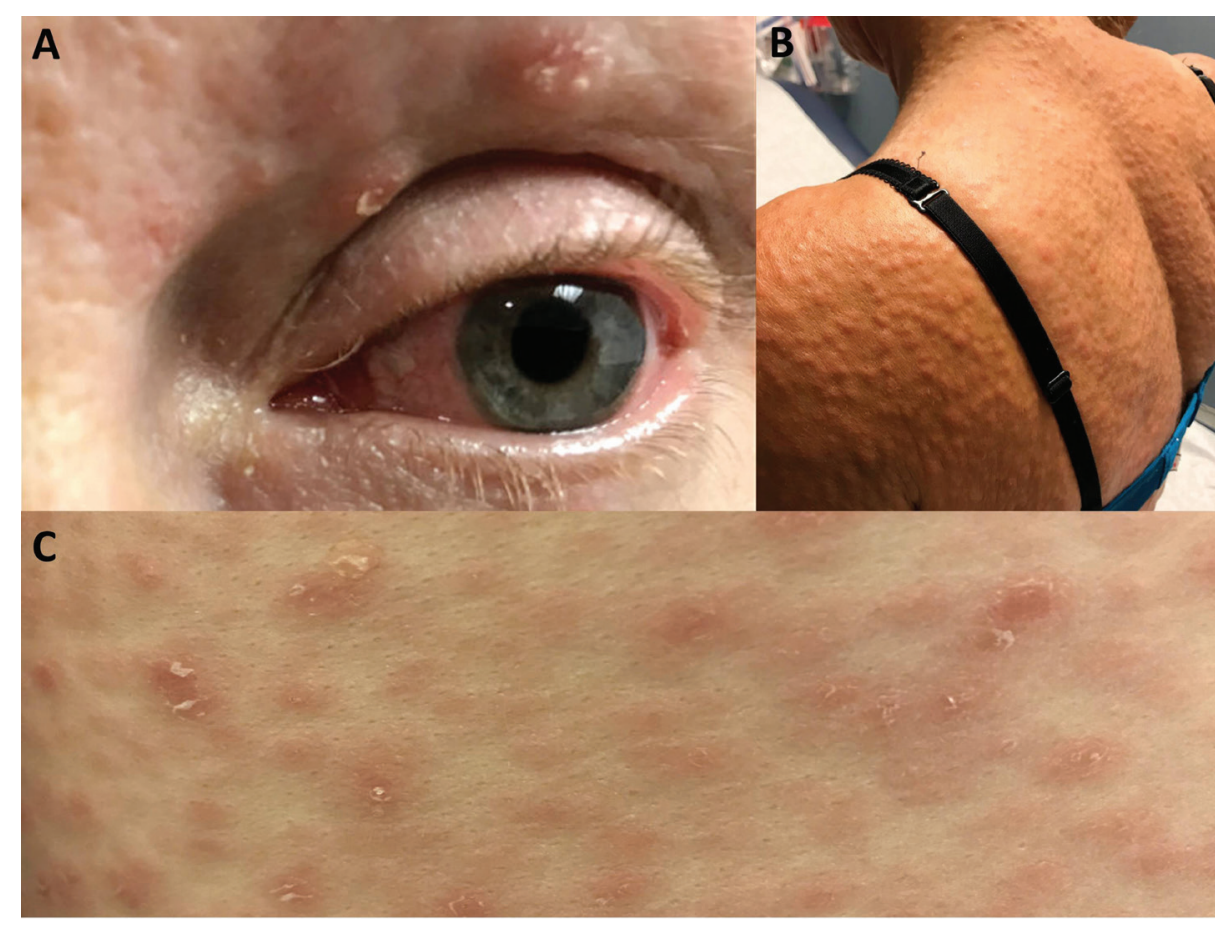

ure 1 : Syphilis secondaire avec neurosyphilis oculaire chez une femme de 42 ans. A) Épiphora (larmoiement accru), injections conjonctivales et œdème périorbitaire. L'examen à la lampe à fente a révélé une uvéite antérieure. B) Éruption papulosquameuse généralisée asymptomatique. C) Papules érythémateuses légèrement squameuses.

Les professionnels de la santé devraient automatiquement soupçonner la présence potentielle d'une syphilis secondaire chez les patients présentant une éruption papulosquameuse généralisée. Lorsqu'un tel diagnostic est possible, ils devraient répéter le test de dépistage de la syphilis, même si un résultat négatif a récemment été obtenu ${ }^{1}$. Un résultat faussement négatif au début de la syphilis primaire peut être dû au fait que la séroconversion se produit après 14-21 jours. II pourrait aussi être dû à l'effet « prozone ", un phénomène par lequel une haute concentration d'anticorps dans un échantillon interfère avec la formation de complexes anticorps-antigène, nécessaires à l'obtention d'un résultat positif². Bien que la neurosyphilis soit généralement considérée comme typique du stade tertiaire, elle peut apparaître à n'importe 
quel stade de la maladie ${ }^{3}$. La patiente devrait subir des tests rapides de la réagine plasmatique 6 et 12 mois après le traitement pour confirmer la guérison complète, ce qui correspondrait à un titre 4 fois moins élevé.

\section{Références}

1. Li L, Cai B, Tao C, et al. Performance evaluation of CLIA for Treponema Pallidum specific antibodies detection in comparison with ELISA. J Clin Lab Anal 2016;30:216-22.

2. Ghanem KG, Ram S, Rice PA. The modern epidemic of syphilis. N Engl J Med 2020;382:845-54.

3. Quilter LAS, de Voux A, Amiya RM, et al. Prevalence of self-reported neurologic and ocular symptoms in early syphilis cases. Clin Infect Dis [Cyberpublication avant impression] 2020 Feb 26;ciaa180. doi : 10.1093/cid/ciaa180.

\section{Intérêts concurrents : Aucun déclaré.}

Cet article a été soumis à l'examen des pairs.

Les auteurs ont obtenu le consentement de la patiente.

Affiliations : Division de dermatologie, Faculté de médecine de l'Université d'Ottawa; Hôpital d'Ottawa, Ottawa, Ont.

Propriété intellectuelle du contenu : Il s'agit d'un article en libre accès distribué conformément aux modalités de la licence Creative Commons Attribution (CC BY-NC-ND 4.0), qui permet l'utilisation, la diffusion et la reproduction dans tout médium à la condition que la publication originale soit adéquatement citée, que l'utilisation se fasse à des fins non commerciales (c.-à-d., recherche ou éducation) et qu'aucune modification ni adaptation n'y soit apportée. Voir : https://creativecommons.org/licenses/by-nc-nd/4.0/deed.fr.

Correspondance : Mark Kirchhof, mkirchho@uottawa.ca 